\title{
Research on The Interaction of Mobile Games
}

\author{
Rao Wei \\ Zhongnan University of Economics and Law, No. 182 Nanhu Avenue, East Lake New Technology \\ Development Zone, Wuhan, Hubei, China
}

10148031@qq.com

Keywords: Mobile game, Interactivity, user, experience

\begin{abstract}
Nowadays, mobile games have become one of the most important ways of people's life and entertainment, and people's enthusiasm for mobile games goes far beyond their obsession with other video game entertainment products and methods. Video games are divided into computer games, television games, mobile games, tablet games, palm-held computers, etc. by the terminal; by type: single-player games, online games, mobile Internet games, virtual simulation games, etc. These games, whether based on hardware technology or network technology, are inseparable from its unique interactive, and mobile games as a branch of electronic games, game design, interactive technology and mobile Internet technology in one, the human-computer interaction throughout. In this paper, the current mobile game collation, analysis of the nature of its interaction, and how to reasonably integrate interactive design thinking into the game are analyzed, so that players can thoroughly feel the interactive experience brought by mobile game, to obtain mobile game benign development.
\end{abstract}

With the popularization and cross-cutting application of electronic technology, digital technology, Internet technology and interactive technology, the development of electronic games has been strongly promoted. Supported by electronic technology and interactive technology, electronic games have been launched: computer games, video games, mobile games, palm-held computers, etc. Supported by digital technology, we have launched: online games, mobile Internet games, virtual simulation games and other products. Mobile games, as an important part of electronic games, make rational use of electronic technology, mobile Internet technology, human-computer interaction and so on, combine games with science and technology, and bring people a strong interactive game entertainment experience. This experience is mainly reflected in the interactive transmission of mobile Internet under the premise of making play. Home can deeply participate in the interaction of video games to a certain extent to create a different game world, so that people feel the experience of mobile games through mobile terminals anytime and anywhere. At the same time, the characteristics of mobile game interaction, such as universality, extensiveness, timeliness and rapidity, enable players to get a better interactive experience. The interactive nature of mobile game is mainly reflected in the interaction between players and mobile phones, players and games, players and players. The interactive nature of mobile game highlights the dual players. Character, the player is not only the experience of the game but also the creator of the interactive environment of the game, which also makes the mobile game with an important user premise, and provides a strong support for its development.

\section{Mobile game interaction analysis}

\subsection{Interactive Classification of Mobile Games}

Interactivity is an indispensable factor in the game. Good interactivity in mobile games is a necessary prerequisite for players to experience the game. If the players in the game because of unreasonable interactivity to bring inconvenience to their own operation or the game does not have an accurate response, this will make the players into the game to a certain extent emotional and swimming. The game creates distance, which also causes the game to lose its meaning and turn to 
other hardware terminal games. Therefore, we must understand what is the interactivity of mobile games, in order to better serve the development of mobile games. The interaction of mobile games mainly includes two parts: interactive mode and interactive content. The interactive mode is what kind of carriers and players can interact with each other, such as using mobile terminals and game software to achieve interaction. Interactive content can be said to include all aspects of the game, including the game's mobile phone interface, screen design, audio-visual effects, game content and so on.

There are many ways of interaction, such as human-computer interaction, human-computer interaction is about the relationship between players and mobile phone hardware and software. In human-computer interaction, various forms are attributed to players and mobile phones. The most prominent feature of human-computer interaction in mobile games is the user's dynamic experience and interactive experience. Such as "subway running cool", "ninja fruit", "talking Tom cat" and so on,_As shown in Figure 1.

In terms of interactive content, there are many types. The interaction between mobile phone users can also be regarded as the communication between game players, especially the mobile game based on a strong group of mobile phone users, so that players have a huge social circle, interpersonal circle, based on which, more game friends are derived to interact. Mobile phone users in the game interaction is not a simple communication between players, the greater interaction is that each participant can directly affect the game perception of other players, which is the biggest highlight of mobile game interaction, but also a new interactive way, so that mobile phone users can be in the same virtual In the virtual world of the game, players interact with each other, which is more practical than human-computer interaction. Therefore, the content of mobile games is also based on the interaction of players, such as "Tencent game", "King Glory", "Jedi Survival" and other games, As shown in Figure 2, 3 . full interaction in mobile games can meet the needs of Gamers'own experience, but also make the psychological expectations of gamers to be realized.

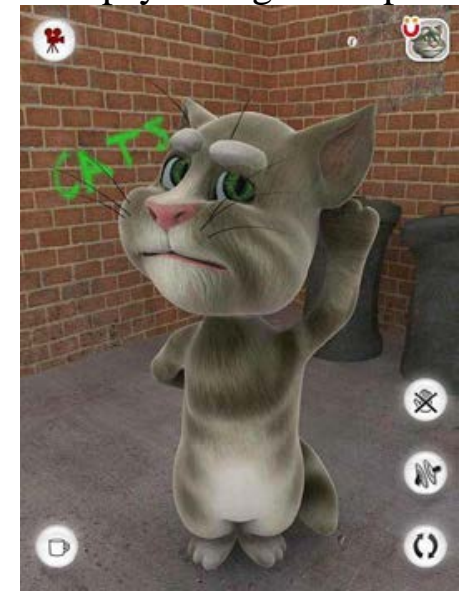

Figure 1: Talking Tom cat

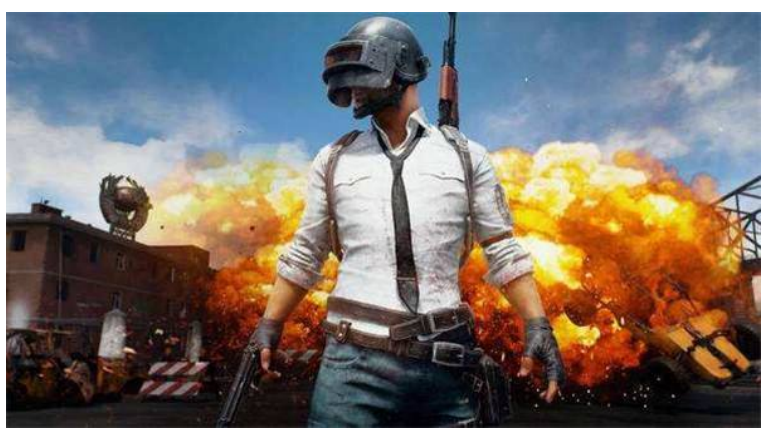

Figure2: Jedi survival - stimulating the battlefield 


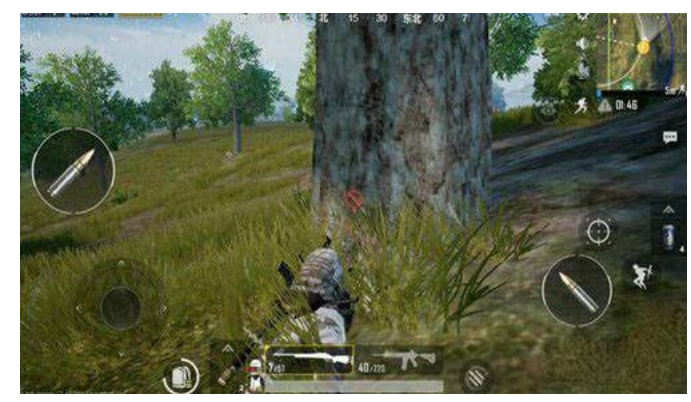

Figure3: Jedi survival - stimulating the battlefield

\subsection{Interactive Communication}

The interactive communication of mobile games can be shown in the information dissemination and interactive activities between players in the game. Many players publish their own or others'game history, game stories on relevant websites, upload photos of players or make video game strategies, etc. This can be seen as between players and game developers. Interactive communication outside the game world. Nowadays, the spread of online games is an invisible way of mass communication. Based on the advantages of network-based media, Online Games integrate mass communication, group communication and interpersonal communication into a whole, so that players form an organizational communication on the game platform, and the relationship between players and their virtual characters is personified. Mobile game combines the characteristics of online games, based on its own mobile Internet advantages, in the game communication mechanism, highlights the mass communication between the disseminator to the game, the organization between disseminators and players, the interpersonal communication between players based on mobile Internet game platform.

There are many ways for mobile game players to co-exist in the interaction of the game. In the design of the game, the selectivity of role-playing is to make players have more sense of existence. This role-playing choice is a bridge between players and the game. These roles have certain authenticity and reflect in the virtual game world. It is characterized by human nature and specific attributes. There are still many interactions in the game, such as language and online and offline interaction of these two kinds of interaction is mainly mobile Internet game is the most prominent. Take " Arena of Valor "as an example, As shown in Figure 4. Players play the role of the game, through interactive operation to achieve the process of the game, players into this huge virtual world, in this virtual world everyone has a different role to play, has different tasks and responsibilities, is a multi-player to build a virtual and real In this environment, players can interact with each other, cooperate with each other and even oppose each other. Among them, the interactive scenes of the game, players can move in the virtual world, in the game is generally interactive narrative, in order to make the players and the game integration, the players through their own operation to promote the development of the game, in the project to promote the plot of the players in the virtual world to communicate, meet each other, arrange. New tasks, especially discussion and communication between players in multiplayer online games, all reflect the interactive spread of mobile games. This also fully reflects the human-computer interaction, the interaction between players, as well as the timeliness of geographical dissemination.

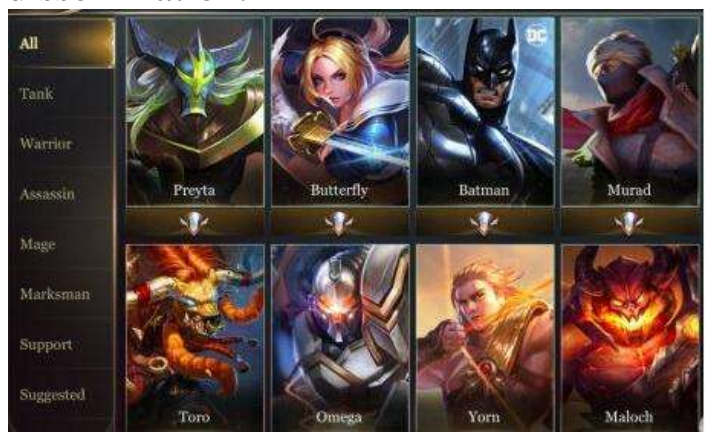

Figure 4 Arena of Valor 


\subsection{Interface Interaction}

Mobile game interactive interface, also known as user interface (UI), refers to the area of human-computer interaction, which exists in all areas of human-computer information exchange, such as the input and output of the mobile screen constitutes this interface. The interaction of mobile phone interface provides a way for players to communicate information with the game itself and form game interaction. Whether the interaction process itself will affect the player's concentration on the content of the game is also a test of the overall artistic characteristics of the game itself. For example, the mobile game "King Glory", a huge and complex story background and game world view, players in the interaction of the game interface will not ignore the experience and feelings of the game story. Players in the game interface experience the aesthetic feeling of the game, is another embodiment of the interface design, is the game itself a key link in the evaluation of the merits and demerits, the rationality of mobile game interface design, determines whether players can better interactive communication. The design of mobile game interface is later than the updating of video game technology. This does not mean that designers can not go beyond the game itself, but the mobile game interface is limited by the technical level, and is limited by the hardware of mobile terminal. With the continuous improvement of electronic technology, the hardware function is becoming more and more powerful, and the degree of artificial intelligence is becoming higher and higher, so that game developers can develop realistic three-dimensional images, and create a virtual reality environment for different users, so that people can feel the game experience brought by the virtual reality world anytime and anywhere.

\section{Mobile game interactive advantages and disadvantages}

\subsection{Mobile Game Interactive Advantages}

The characteristics of mobile phone interaction platform are reflected in the following aspects: (1) mobile terminal interaction. One of the most common ways of human-computer interaction, based on the traditional mobile phone user operation, allows players to touch the mobile phone interface in the game. (2) mobile Internet platform interaction. However, unlike stand-alone mobile games, through mobile Internet platform, more mobile phone users will be linked up in series, through this time-sensitive and special interactive way at any time and anywhere, so that players experience different game feelings and game psychology. (3) mobile phone single machine intelligent interaction. With the development of science and technology, players have a better mobile terminal, highlighting its interactive experience personalized, intelligent, comprehensive, and even through virtual simulation technology, so that this interactive experience more authentic.

The advantages of mobile game interaction are mainly reflected in the following aspects: (1) interface interaction. Mobile game interface is different from computer or other terminals, which means that the touch is made by visual information on a limited screen. In the game, images, text, interface layout, operation layout, visual angle layout, information reception and so on will affect the human-computer interaction experience of gamers. (2) role interaction. Characters are the main way for players to enter the game world. Through the control of characters, players gradually integrate themselves into the game world as the game progresses. (3) environmental interaction. Game environment refers to the virtual world in the game. Game environment is a platform for players to communicate and interact. A good game environment can bring better comfortable experience and feeling, better let players into the game state, to achieve harmony between players and characters, players and players. (4) sound interaction. Sound is the most direct contact between players and the game, the appropriate sound efficiency of the game to better convey the atmosphere and emotion of the game to the player, so that the player with the sound fully feel the experience of the game. (5) Content Interaction: Game content interaction is the core of the game, the player according to the game content to make specific operations, and at the same time because of the content to make the body response, prompting the player to respond, forming a good human-computer interaction. 


\subsection{Mobile Game Interaction Disadvantages}

Although mobile games have many advantages, mobile games are different from computer games or other electronic terminal games. The interactive experience of electronic games is mainly expressed in the aspect of "playing". However, the interactive experience of mobile games is questioned. Because of the limitations of terminal devices, many games are embodied. Out of the "play", this video game can not be reflected in the mobile terminal, which is also a lot of computer games can not coexist in the mobile terminal reasons. For example, in 2017, the world's hottest "chicken eating" game, accounting for half of the computer terminal, the major game manufacturers and platforms have launched similar "chicken eating" mobile phone games, not as hot as the computer terminal, although with a considerable number of mobile phone players, but in the game experience and playability of players with electricity There is still a big gap between brain terminals. How to solve the playability of mobile games, which is also a major problem facing mobile game developers.

Nowadays, there are many low-headed people in social life. Because of the characteristics of mobile terminal and the advantages of platform, many mobile game players spend a lot of time on the game, forming an autistic state, which can be regarded as players into addicted state. Game addiction will make players tend to be indifferent to the real world and lose their feelings. Participation and feeling consciousness, long-term addiction to games, will lead to people's lack of social experience, as well as the decline of social adaptability, but also to the player's health hazards. Therefore, mobile game interaction design needs to take into account the balance between the game and the player, to avoid players indulging in uncontrolled games, such as age limits or game time settings, can effectively control the player to spend a lot of time in the game, while interactive design also needs to consider the game balance, to avoid causing players to be bored with the game. Depression and other emotions. The interaction of the game environment will also have an impact on the player, uncontrolled pornography, violent games, will mislead the player's psychology, physical and mental harm.

\section{Interaction needs and expectations of mobile game users}

\subsection{Interactive needs of mobile game users}

(1) entertainment and interaction. Human-computer interaction is an important factor in the game, but also the root of the existence of the game, different games bring to the game players experience is also different. Game players are eager to integrate into the virtual world of the game, and with the development of the game plot to get a complete game experience. Of course, in the process of experiencing the game, there are many components of human-computer interaction, in another way, the interaction of the game to a greater extent enhance the playability.

(2) independence and imagination. Players in the game, the player and the mobile game between the formation of an independent virtual space, the player in this virtual space as a participant, leader, role playing. Players can carry out each game task or level according to the mode defined by the game itself. Players can also play the game according to their imagination, so as to get different game experience. Different kinds of games bring different feelings to gamers and meet the needs of different groups of people, such as "My World" to gamers to bring a space of imagination, as shown in Figure 5, And "Stone Legend" to bring a static mental game, as shown in Figure 6.

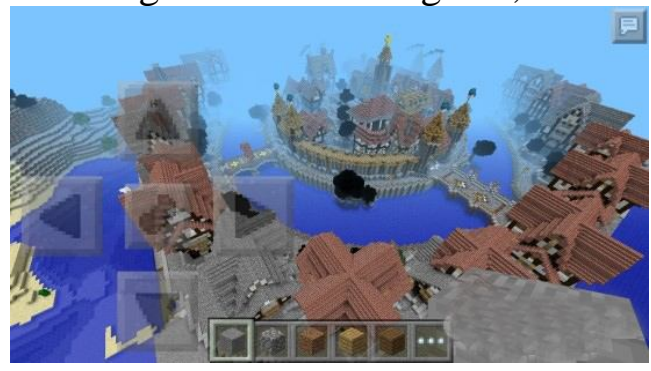

Figure5: My World 


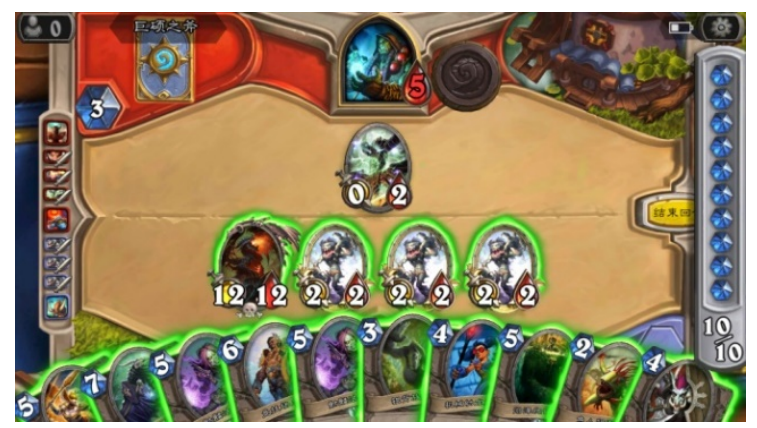

Figure6: Stone Legend

\subsection{Mobile game users' interaction expectations}

(1) the feasibility of operation. The world in the game is another copy of the real world. In the virtual game environment, players can judge which operations are feasible and what results will be caused by the experience of real life. These operations are expected by the player, and desire to skilled operation, only in this way can players truly experience the entertainment of mobile games. Therefore, these operations must be implemented in the mobile game interface, Such as mobile phone game " Catch the fish ", as shown in Figure 7.

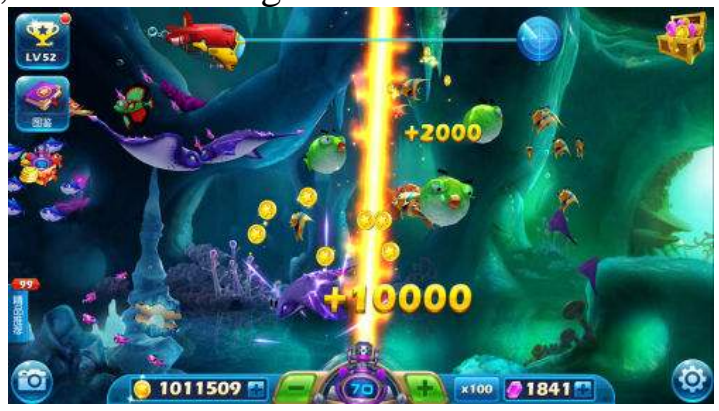

Figure7: Catch the fish

(2) the guidance of the game interface. Game can be compared to a complete story, the player in the virtual world in the game, experience the story. Players in the game continue to complete the task, through the checkpoint, in the process of the game depend on their own step by step exploration, until the completion of the game. No matter what type of mobile games, players hope that the interface in the game interface can be assisted by guidance, at this time, mobile game interface needs to highlight the role of assistance and guidance in the limited screen range. Therefore, the interface design of mobile games should be categorized and clear, and become the guide for players to complete the game smoothly, Such as mobile phone game " Crazy speed ".

\section{Conclusion}

With the development of science and technology, more and more technologies, such as human-computer interaction, artificial intelligence, mobile Internet technology, digital technology, virtual simulation technology, are applied to mobile games, so that people have more interaction with mobile phones, but also greatly expand the freedom of interactive video games. The multi-interaction of mobile games reflects the future development trend of human-computer interaction. The game development industry is also moving towards the direction of multi-interaction and experience. It integrates the elements of the real world into mobile games and brings more real experience to the players. Of course, game vendors are aware of the drawbacks of existing mobile game interactivity, artificial intelligence, virtual reality and other ideas are also working hard to achieve, I believe in the near future, mobile games will bring us more and better interactive experience, will become one of the most important means of information dissemination and interactive activit. 


\section{References}

[1]Zhang Yi's "interactive research on contemporary video games" [J], world garden 2013 eighth issue.

[2] Wu Jingwen, Li Fangfen, On the Impact of Interactive Technologies on Video Games and Future Development [J], [Technological Innovation and Application] Issue 12, 2013.

[3] Han Shuai "interaction, immersion and aesthetics in electronic travel" [J], industry and Technology Forum 2011.

[4] Meng Wei, Interactive Communication in Video Games: An Analysis of Gamers in Games [J], Henan Social Sciences Volume 16, No. 3, May 2008.

[5]Ren Xiaomin, Research on Computer Game Interface Design Based on Human-Computer Interaction [J], Electronic Production, Issue 5, 2013. 